[Aus dem Seemannskrankenhaus und Institut für Schiffs- u. Tropenhygiene.] (Direktor: Med.-Rat Prof. Dr. Nocht.)

\title{
Vergleichende \\ bakteriologische Blut-, Stuhl- und Urinuntersuchungen bei Typhus abdominalis.
}

Von

Dr. Albert Bohne, Sekundärarzt.

Von dem reichen Typhusmaterial des Seemannskrankenhauses habe ich eine Anzahl von Fällen systematisch untersucht, indem ich mir die Frage vorlegte, welche Methode uns am zuverlässigsten, schnellsten und einfachsten zu einer Typhusdiagnose verhilft. Ich verfolgte dabei nur praktische Ziele und verzichtete daher, wenn eine Methode mir den Nachweis erbrachte, weiter zu forschen, nach welcher Zeit die anderen ein Resultat lieferten. Bei allen Fällen wurden Blut, Stuhl und Urin verarbeitet, und zwar kamen folgende Methoden zur Anwendung.

I. Für Blut.

1. Die bekannte Schottmüllersche Methode: $1 \frac{1 / 2}{\text { bis }} 2^{\mathrm{cem}}$ Blut werden in einem Kolben mit $100 \mathrm{cem}$ Bouillon verdünnt (B). ${ }^{1}$

2. Die Conradische Gallenanreicherungsmethode: $10^{\mathrm{com}}$ des Peptonglyzeringallengemisches werden mit 2 bis $5 \mathrm{ccm}$ Blut versetzt (K). Das Gemisch besteht aus Rindergalle mit 10 Prozent Pepton und 10 Prozent Glyzerin.

3. Die Meyersteinsche Anreicherung mittels konzentrierter Gallensalze: 2 bis $3^{\mathrm{cem}}$ Blut werden mit je 2 bis 3 Tropfen der Gallensalze vermischt und gut durchgeschüttelt (M).

1 Die in Klammern gesetzten Buchstaben dienen als Abkürzungen für die Tabelle und den weiteren Text. 
Übersichts-

\begin{tabular}{|c|c|c|c|c|c|c|c|c|c|c|c|c|c|c|}
\hline 总 & & & & & 点 & 节 & $\begin{array}{r}\text { Fickers I) } \\
\text { am } \mathrm{T}\end{array}$ & $\begin{array}{l}\text { nostikum } \\
\text { der }\end{array}$ &.$\infty$ & 姁 & & 量 & & $\stackrel{50}{g}$ \\
\hline 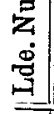 & $\mathrm{N}$ a m & $\theta \mathrm{n}$ & & & 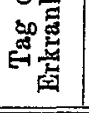 & 苞 & $\begin{array}{c}\text { a) } \\
\text { Unter- } \\
\text { suchung }\end{array}$ & $\begin{array}{c}\text { b) } \\
\text { später }\end{array}$ & 容 & 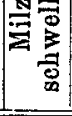 & 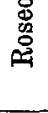 & 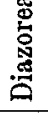 & 㞼 & 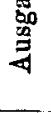 \\
\hline 1 & Desens . & & . & & 22. & 38.2 & $1: 100$ & $1: 100$ & - & + & - & - & V. & \\
\hline 2 & Fimmel . & 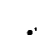 & . & & 9: & 39.8 & $1: 50$ & $1: 100$ & + & + & + & + & D. & \\
\hline 3 & Halfardson. & . & . & & $10 \%$ & $39 \cdot 0$ & $1: 100$ & $1: 100$ & + & - & + & - & V. & \\
\hline 4 & Condellis & • & • & & $?$ & 38.0 & $1: 100$ & & + & - & - & - & V. & \\
\hline 5 & Meyer . & $\cdot$ & . & & 24. & $39 \cdot 0$ & $1: 100$ & & - & - & - & - & D. & \\
\hline 6 & Kruse . : & . & . & & & & & & & & & & & \\
\hline 7 & Nielson . & . & - & & & & & & & & & & & \\
\hline 8 & Hogberg . & 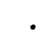 & . & & & & & & & & & & & \\
\hline 9 & Krogh . . & & . & • & & & & & & & & & & \\
\hline 10 & Kalita . & 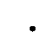 & - & 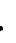 & & & & & & & & & & \\
\hline 11 & Martinsen . & . & . & • & & & & & & & & & & \\
\hline 12 & Vries. . . & 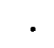 & - & & 22. & $39 \cdot 5$ & $1: 100$ & & - & + & - & + & D. & \\
\hline 13 & Drees . & 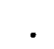 & . & & 9. & 39.5 & 1: 50 & $1: 100$ & - & + & + & + & V. & \\
\hline 14 & Schulz . & 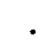 & - & & 12. & 39.5 & 1: 50 & $1: 100$ & + & + & + & + & D. & \\
\hline 15 & Zander. & . & • & & 7. & 39.0 & - & $1: 100$ & - & + & - & + & D. & \\
\hline 16 & Johaminson & $\cdot$ & . & & 9. & 40.0 & - & $1: 100$ & + & + & - & + & D. & \\
\hline 17 & Albrecht . & . & . & • & 15. & $40 \cdot 0$ & - & - & + & + & + & + & V. & $t$ \\
\hline 18 & Wrzinski & . & . & • & 15. & $39 \cdot 0$ & - & $1: 100$ & + & + & + & - & V. & \\
\hline $1 \dot{9}$ & Regensdorf. & . & . & & 11. & $40 \cdot 1$ & $1: 100$ & & + & + & + & - & V. & $t$ \\
\hline 20 & Thams. & .. & . & & 32. & $39 \cdot 0$ & $I: 100$ & & + & + & + & + & D. & \\
\hline 21 & Neetz . & . & . & & 16. & $39 \cdot 5$ & 1: 50 & $i: 100$ & + & + & + & + & D. & \\
\hline 22 & Renhvall . & - . & . & & 16. & 39.0 & $1: 100$ & & + & + & + & + & D. & \\
\hline 23 & Turban . & . . & . & & 10. & 40.0 & $1: 100$ & & + & + & + & + & V. & $t$ \\
\hline 24 & Dreesmann. & - . & . & & 11. & $40 \cdot 0$ & & & + & + & + & + & V. & $t$ \\
\hline 25 & Smith . & . . & . & & 15. & 39.0 & $1: 100$ & & - & + & + & - & D. & \\
\hline 26 & Wolf . . & . . & - & & 15. & 38.7 & $1: 100$ & & 一 & + & + & - & V. & \\
\hline 27 & Healy : . & - : & - & & 11. & $40 \cdot 0$ & $1: 100$ & & + & + & + & - & V. & \\
\hline 28 & Kempner . & . . & - & & 19. & 39.2 & $1: 100$ & & - & + & + & - & V. & \\
\hline 29 & Butters - . & $\cdot \cdot$ & - & & 9. & $39 \cdot 0$ & - & $-1: 100$ & - & + & + & - & V. & \\
\hline 30 & Mierke . . & ${ }^{\circ}$ & . & & 11.(?) & 40.0 & $1: 100$ & & + & + & + & + & V. & \\
\hline 31 & Torgessen . & . . & . & & 32. & 39.0 & $1: 100$ & & - & + & + & + & V. & \\
\hline 32 & Schneider. & . . & - & & 6. & $39 \cdot 8$ & 1: 50 & $1: 100$ & + & + & + & + & D. & \\
\hline 33 & Möser . . & $0^{\circ}$ & . & & 11. & $39 \cdot 5$ & $1: 100$ & & - & - & - & + & D. & \\
\hline 34 & Quenzel. & . . & - & & 23. & $39 \cdot 0$ & 1: 50 & $1: 100$ & + & + & + & + & D. & \\
\hline 35 & Leiss. . . & . . & - & & 9. & 40.0 & $1: 100$ & & + & + & + & + & V. & \\
\hline
\end{tabular}


tabelle.

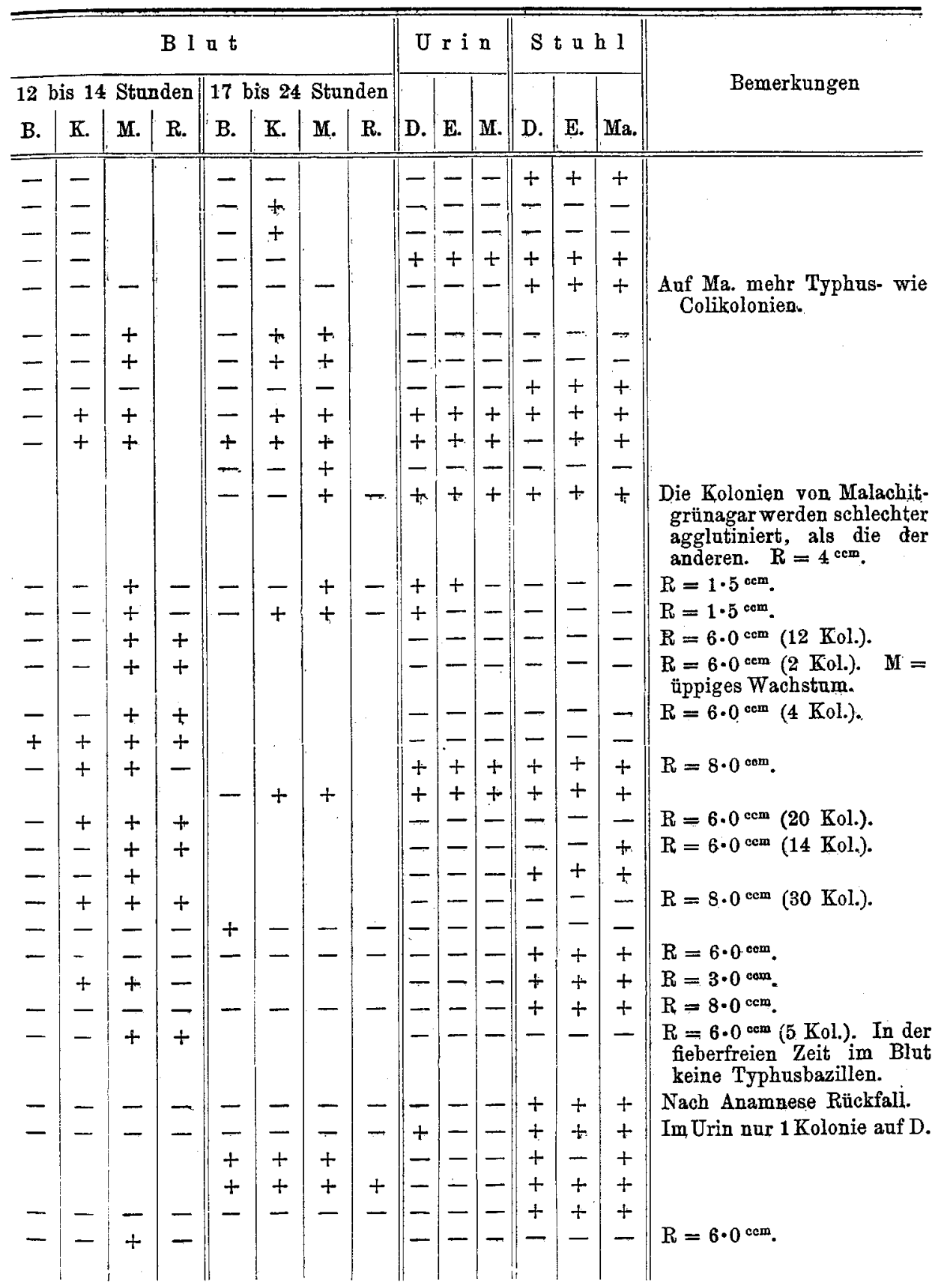


4. Gewöhnlicher Agar, dem nach Rosen-Runge 1 Prozent Natrium glycocholicum zugesetzt war. Auf jedes Röhrchen mit 10 bis 15 com kommen $2^{\mathrm{com}}$ Blut (R).

Außerdem vermischte ich in einigen Fällen das nach Meyerstein behandelte Blut mit gewöhnlichem Agar und goB dann Platten. Da die Methode aber keine befriedigenden Resultate gab, habe ich sie bald wieder aufgegeben.

Von den unter 1. bis 3 . hergestellten Mischungen wurden meist nach 12 bis 14 Stunden Ausstriche auf Drigalski oder gewöhnlichem Agar angefertigt; bei negativem Ausfall wurde es nach 24 Stunden wiederholt. $\mathrm{DaB}$ die sämtlichen Blutuntersuchungen an einem Tage gleichzeitig ausgeführt wurden, bedarf wohl keiner besonderen Erwähnung.

\section{Für Stuhl and Urin:}

Es kamen zur Verwendung Drigalski- (D), Endo- (E) und Malachitgrünplatten (M) nach den Angaben von Leuchs. Die Platten wurden meist am Tage der Blutuntersuchung angefertigt, nur ausnahmsweise 24. Stunden später.

Über das Ergebnis meiner Untersuchungen gibt die Übersichtstabelle Auskunft.

Bevor ich das Ergebnis der vorstehenden Tabelle bespreche, ist es notwendig, mit einigen Worten auf die Zusammensetzung unseres Materials einzugehen.

Wie aus der ersten Rubrik der Tabelle ersichtlich, kommen unsere Patienten in der 1. Woche ihrer Erkrankung nur selten zu uns. Meist erfolgt die Infeltion in den nordamerikanischen oder südamerikanischen Hāfen und auf der Heimreise kommt dann die Krankheit zum Ausbruch. Nur selten sind die Patienten imstande, genau den Beginn ihrer Erkrankung angeben zu können, so daB die Zahlen der ersten Rubrik nicht als einwandfrei gelten können. So machte z. B. der Patient Mirke, ein 16 jähriger Steward, folgende Angaben über die Vorgeschichte seiner Erkrankung: Einen Monat vor seiner Aufnahme in das Seemannskrankenhaus sei er mit Fieber erkrankt, das 14 Tage anhielt, und mit Chinin behandelt wurde. Er war dann 5 Tage wieder arbeitsfähig und erkrankte erst wieder 9 Tage vor Hamburg. Ich bespreche diesen Fall etwas genauer, weil er auf die Deutung der Resultate nicht ohne Einfluß ist. Nach allem kann man aber wohl sagen, daB die Zahlen der ersten Rubrik eher zu niedrig als zu hoch angegeben sind.

Die nunmehrige Besprechung meiner Ergebnisse soll in der Weise erfolgen, daB für die einzelnen Krankheitswochen zunächst das Resultat der Blutuntersuchung mit denen von Stuhl und Urin verglichen werden 
soll. Im weiteren werden die verschiedenen Methoden bei jedem der drei untersuchten Medien gegeneinander abgewogen werden. Um Wiederholungen zu vermeiden, sollen die einzelnen Methoden nur mit den obengenannten Buchstaben bezeichnet werden.

In der 1. Woche kamen nur zwei Fālle zur Untersuchung. Bei beiden ergab die Blutuntersuchung eine positive, die Stuhl- und Urinuntersuchung ein negatives Resultat.

Von den verschiedenen Methoden der Blutuntersuchung war

B positiv in 50 Prozent,

K " " 50 , ,

M positiv in 100 Prozent,

Bei den 13 Fällen der 2. Woche wurden im Blut 12 mal, im Stubl 3 mal, im Urin 5 mal Typhusbazillen nachgewiesen. Der negative Fall der Blutkultur betraf den oben ausführlich besprochenen Patienten Mirke. Bei der Blutkultur gaben die 13 Fälle der 2. Woche nach einer Anreicherung von 12 bis 14 Stunden bei:

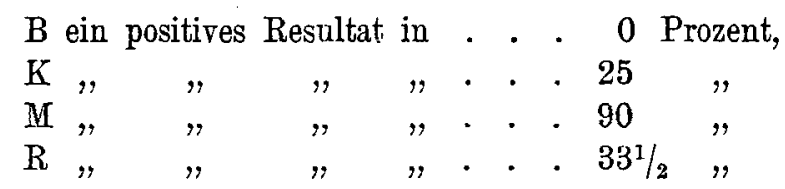

Von 7 Patienten der 3. Woche hatten Typhusbazillen im Blut 4, im Stubl 3, im Urin 0. Bei der Blutuntersuchung war positiv nach einer Anreicherung von 12 bis 14 Stunden:
$B$ in $14 \cdot 3$ Prozent,
$\mathrm{K}, 28 \cdot 6, "$,
M in 57.2 Prozent,
$\mathbf{R}, \mathbf{5 7 . 2}$, .

Der 4. Woche gehörten vier Patienten an. Bei ihnen war der Bazillenbefund positiv im Blut in einem Fall, im Stuhl in vier Fällen, im Urin in einem Fall. Den einzigen positiven Blutbefund hatte $\mathrm{M}$ nach 24 Stunden geliefert, während $B, K$ und $R$ kein Wachstum zeigten.

Die 5. Woche mit einem Patient ergab eine positive Blutkultur. Stuhl und Urin enthielt in beiden Fällen Typhusbazillen. Die Blutkultur war positiv bei $\mathrm{K}$ und $\mathrm{M}$.

Aus den Zahlen geht wohl ohne weiteres hervor, daß die Anreicherung nach Meyerstein allen anderen Methoden an Zuverlässigkeit, Schnelligkeit und Einfachheit überlegen ist. Sie wird um so sicherer zum Ziele führen, je früher der Patient zur Untersuchung gelangt. Bei der Verwendung der verschiedenen Nährböden erlebte ich zuerst eine Enttäuschung. Ich hatte sehen können, wie vorzügliche Resultate $\mathrm{Hr}$. Dr. Leuchs mit seinen Malachitgrünnährböden im Laboratorium erzielt hatte, und ging mit großen Erwartungen an die Utbertragung in die 


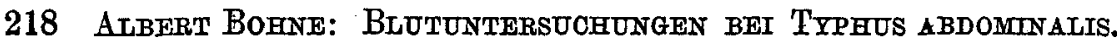

Praxis. Bald aber machte ich die Erfahrung, wie andere vor mir, daB die verschiedenen Typhusstämme sich sehr verschieden verbalten. Während in dem einen Fall (5) Coli gehemmt und Typhus begünstigt wurde, war bei den meisten anderen das Gegenteil der Fall. Noch einen anderen. Nachteil hat der Malachitgrünnährboden gegenüber dem Endoschen und Drigalski-Nährboden. Bei der orientierenden Agglutination nämlich werden die Kolonien von Malachitgrünnährboden hänfig sehr viel schwerer agglutiniert als die von den beiden anderen herrührenden Kolonien. So trat bei der Verwendung von Mala,chitgrünnährböden die Agglutination nicht selten erst bei einer Verdünnung von 1:100 auf, während bei den anderen Nährböden selbst eine Verdünnung von 1:500 noch momentane Agglutination erzeugte. Schließlich sind auf den Nährböden nach Endo und Drigalski auch für den weniger Geübten die Typhuskolonien leichter zu unterscheiden, so daß für die Anlegung von Stuhlplatten die Verwendung ron Enđo- und Drigalski-Nährböden vorzuziehen ist. Dem Drigalski-Agar gegenüber hat der Endo-Agar noch den Vorzug der einfacheren Herstellung voraus.

Das Ergebnis meiner Untersuchung kann ich zusammenfassen in folgende Sätze:

1. Die Gallenanreicherung nach Meyerstein ist die einfachste, sicherste und schnellste.

2. Die Blutkultur ergibt um so häufiger positive Resultate, je früher sie ausgeführt werden kann.

3. Für Reinzüchtung aus Stuhl und Urin ist zurzeit noch der Endound Drigalski-Agar dem Malachitgrünagar vorzuziehen.

\section{Literatur-Verzeichnis.}

1. Conradi, Münchener med. Wochenschrift. 1906. Nr. 2, 34 u. 49.

2. Meyerstein, Ebenda. 1906. Nr. 38 u. 44.

3. Rosen-Runge, Centralblatt für Balteriologie. Orig. Bd. XLIII.

4. Kayser, Ebenda. Orig. Bd.XLII. - Münchener med. Wochenschrift. 1906. Nr. 17 a. 40.

5. Leuchs, Deutsche med. Wochenschrift. 1906. Nr. 33.

6. Löffler, Ebenda. 1903. Nr. 36. 